\title{
FEM Approximation of Internal Combustion Chambers for Knock Investigations
}

\author{
Sönke Carstens-Behrens, Mark Urlaub, and Johann F. Böhme \\ Ruhr Universität Bochum \\ Jürgen Förster and Franz Raichle \\ Robert Bosch $\mathrm{GmbH}$
}

Copyright (C) 2002 Society of Automotive Engineers, Inc.

\begin{abstract}
The resonances of SI engine combustion chambers are slightly excited during normal combustion but strongly excited by knock. In order to avoid knocking combustions extensive knowledge about knock and its effects is necessary. In this paper the combustion chamber of a serial production engine is modeled by finite elements. Modal analyses are performed in order to gain information about the resonances, their frequencies, and their frequency and amplitude modulations. Simulation results are compared to measured data using a highresolution time-frequency method. Furthermore, a connection between knock origin and the excitation of the resonances is postulated applying transient analyses.
\end{abstract}

\section{INTRODUCTION}

Although the appearance of knocking combustions and their problems concerning pollutant production, engine efficiency, and engine damages have been investigated for decades $[1,2]$, there are still many aspects that are not completely understood and more knowledge would be helpful. This holds for the primary effect, the chemical processes of the combustion, as well as for the secondary effect, the excitation of the pressure resonances of the combustion chamber, as well as for the tertiary effect, the transfer of the resonances through the engine housing as structure-borne sound. This work mainly addresses the secondary effect, the excitation of the pressure resonances by knock. Since measuring pressure in a combustion chamber is relatively feasible compared to e.g. optical measurements of the flame front propagation, pressure signals have become the most important information source for knock investigations. It is well known that the pressure resonances are standing waves in the combustion chamber exhibiting pressure nodes and antinodes. Therefore the pressure amplitude for each resonance depends on the considered location. Since pressure can not be measured at many locations inside an operating cylinder, the resonances must be modeled. Draper [1] estimated the combustion chamber resonances of an aircraft engine by solving the wave equation for a right cylindrical resonant cavity. With decreasing combustion chamber volume this approximation is too simple. Hickling et al. [2] reported on the additional influence of the cylinder head that causes a mode splitting, i.e. a resonance may appear twice, rotated by a certain angle and with different frequency. They also mentioned the application of the finite element method (FEM) for modeling the combustion chamber. Scholl et al. [3] used this approach to investigate the combustion chamber resonances of a two- and a fourvalve cylinder. They considered a crank angle interval from $10^{\circ}$ to $95^{\circ}$ degrees and compared the simulation results to measured data using the spectrogram as timefrequency representation. This way they proved that FEM is a useful tool in order to model more realistic combustion chamber geometries. They demonstrated the mode splitting and mentioned the problem of observing pressure resonances because sensors might be located at pressure nodes.

This work extends the investigations of Scholl et al. with respect to following aspects:

- The considered crank angle interval covers the whole range from $0^{\circ}$ to $180^{\circ}$.

- Compared to the spectrogram a time-frequency representation with better resolution is applied so that simulated and measured data can be compared more accurately.

- In addition to the frequency modulation an amplitude modulation is observed and described.

- A sensor characteristic is presented to predict the signal obtained by a pressure sensor depending on its position.

- For the first time transient simulations were performed in order to simulate the explosive character of knock. That way a possible influence of knock origin on the excitation of the resonances can be studied. 


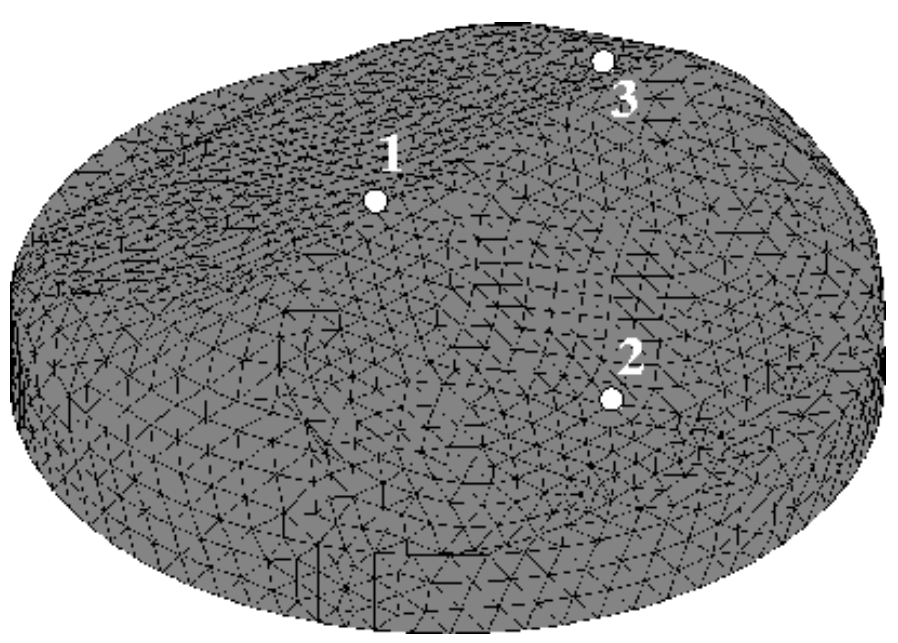

Figure 1. FEM model of the combustion chamber at a piston position of $10 \mathrm{~mm}$ and the positions of three virtual pressure sensors.

\section{MODELING}

The investigations base on a spark ignition engine with four valves, a central spark plug, and a capacity of circa $0.42 \mathrm{l}$ per cylinder. The combustion chamber of this engine was modeled using the commercial FEM package ANSYS. The element FLUID30 was used to model the combustion chamber gas as fluid with zero viscosity. The chamber walls were considered to be absolutely rigid, reflections at the chamber walls are lossless. The speed of sound was set constant to $1000 \mathrm{~m} / \mathrm{s}$ for all simulations. For comparison with measured data the speed of sound can be corrected afterwards. The crank angle interval covered the whole range from $\alpha=0^{\circ}$ to $\alpha=180^{\circ}$. This corresponds to a piston position range from $z=0 \mathrm{~mm}$ to $z=82.7 \mathrm{~mm}$. Simulations were performed with a piston position step size of $\Delta z=0.2 \mathrm{~mm}$. Actually the piston moves continuously but this quasi-static approximation is justified because the speed of sound is much faster than the piston velocity, [3]. Simulation results will also confirm this assumption. For meshing, the combustion chamber volume was divided into two volumes: the cylinder head volume, constant for all simulations, and the piston volume that depends on piston position. The cylinder head volume was meshed in the same way for all simulations while the piston volume mesh changed depending on piston position. For all simulations the mean node distance was approximately $3 \mathrm{~mm}$. This was the finest mesh that could be simulated by the hardware at bottom dead center. Using modal analyses a frequency range from $1 \mathrm{kHz}$ to $27 \mathrm{kHz}$ was searched for resonances at each piston position. Figure 1 shows the combustion chamber model at a piston position of 10 $\mathrm{mm}$. Furthermore, three nodes are marked that will serve as virtual pressure sensors in order to compare their signals with those of real measurements. Sensor 1 could be mounted in a central spark plug. Its position is slightly decentralized from the cylinder axis. Sensor 2 and 3 could be mounted in the cylinder head.

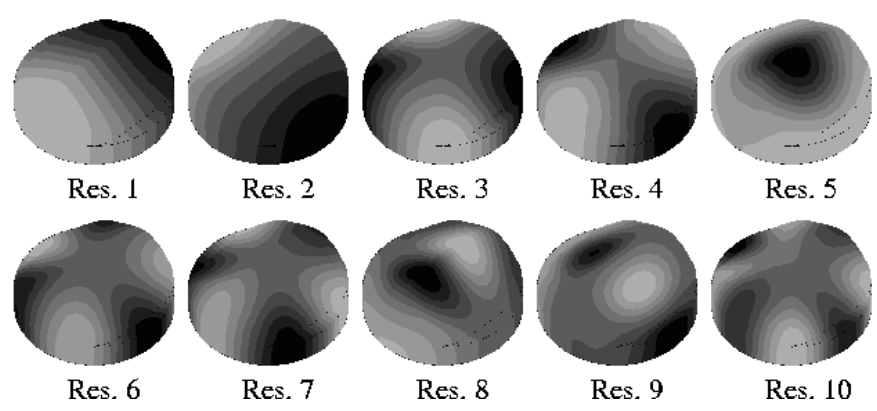

Figure 2. Pressure distribution of the first ten resonances at a piston position of $10 \mathrm{~mm}$.

\section{METAMORPHOSIS}

Figure 2 shows the shape of the first ten resonances found at a piston position of $10 \mathrm{~mm}$. Bright regions mean high pressure, dark regions mean low pressure. Notice that the figures only show a time instant of each oscillation, at a later instant corresponding to a phase shift of $180^{\circ}$, high and low pressure would be exchanged. Only the mean pressure indicated by mean gray does not change because it marks pressure nodes. Res. 1 and 2 demonstrate the mode splitting reported by Hickling et al. They have a very similar shape but they are rotated by an angle of $90^{\circ}$. Because of the influence of the cylinder head they have different frequencies: $6.3 \mathrm{kHz}$ and $7.5 \mathrm{kHz}$, respectively. Res. 3 and 4,6 and 7 , and 8 and 9 are also subjected to mode splitting, but their frequencies are closer together. For each piston position or crank angle, respectively, the shape of the resonances is fixed. But for different piston position the shape of most resonances changes, see Figure 3 . While the shape of the first and third resonance do almost not change, the other resonances run through a metamorphosis. For example, Res. 2 and Res. 4 seem to exchange their pressure distribution between piston position $60 \mathrm{~mm}$ and $70 \mathrm{~mm}$. But the piston position step size is too large in this figure in order to understand the metamorphosis. Therefore, Figure 4 shows the piston position range of interest. Here it is visible how the antinodes tip over. The metamorphosis of the other resonances can be explained by the same way. Because of the metamorphoses it was necessary to have a small simulation step of $0.2 \mathrm{~mm}$ between two piston positions. In the considered frequency range between 15 and 70 resonances were found depending on piston position. This means that more than 10000 resonances had to be classified to the 70 possible resonances. This enormous work was done using a pattern recognition algorithm that took into account both the pressure distribution and the frequencies of the resonances.

\section{FREQUENCY MODULATION}

Draper has already noticed that the frequencies of the resonances are modulated. There are two reasons for the modulation: (a) The speed of sound of the gas 
Res. 1
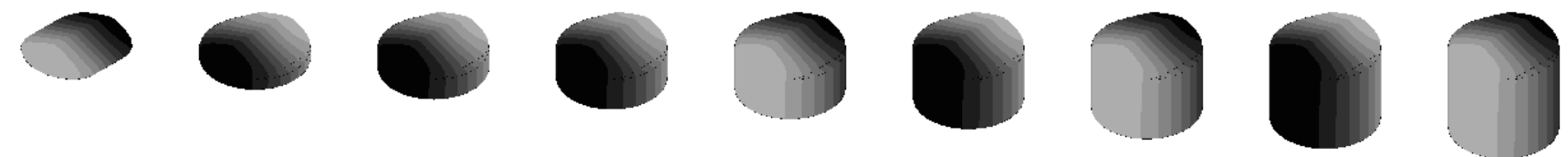

Res. 2
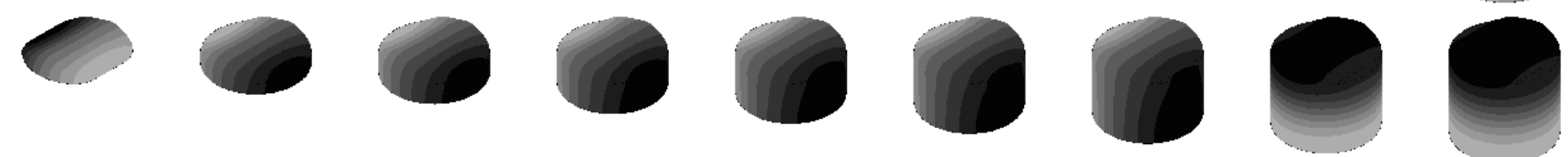

Res. 3
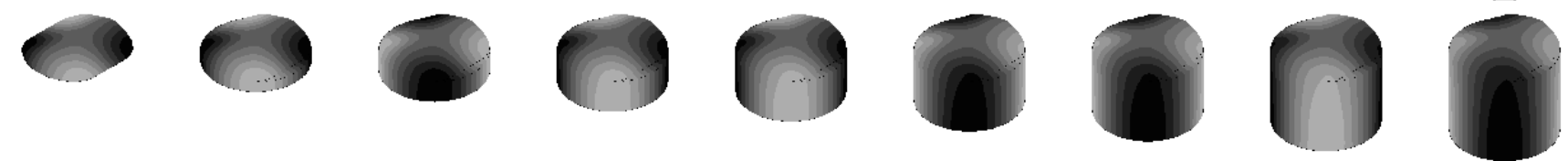

Res. 4
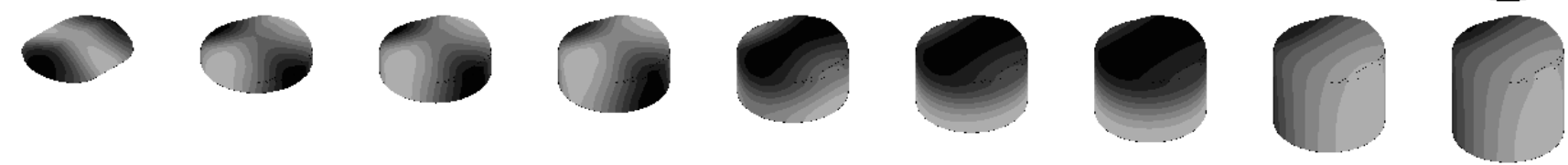

Res. 5
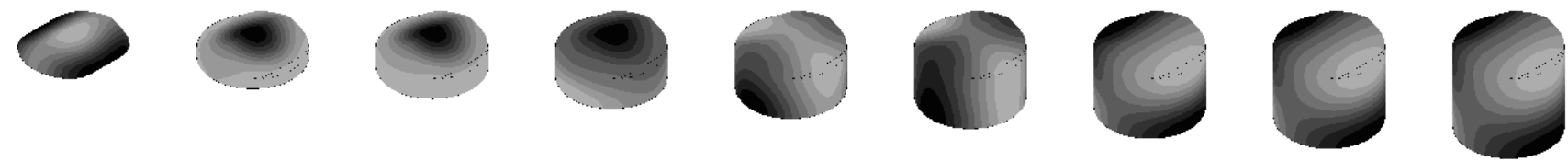

$0 \mathrm{~mm}$

$10 \mathrm{~mm}$

$20 \mathrm{~mm}$

$30 \mathrm{~mm}$

$40 \mathrm{~mm}$

$50 \mathrm{~mm}$

$60 \mathrm{~mm}$

$70 \mathrm{~mm}$

$80 \mathrm{~mm}$

Figure 3. Pressure distribution of the first five resonances depending on piston position.

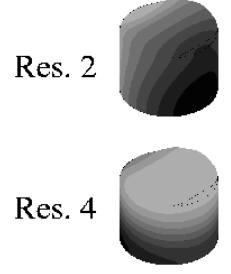

$67.4 \mathrm{~mm}$
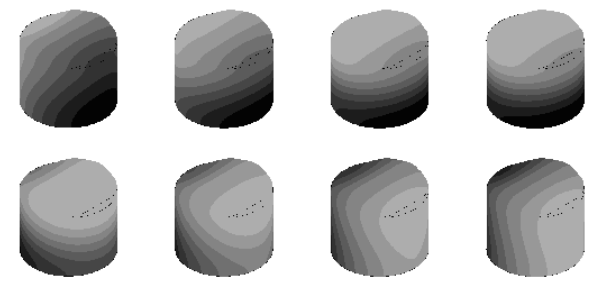

$67.8 \mathrm{~mm}$
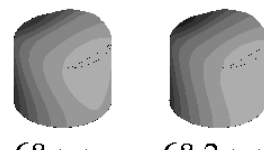

$68.2 \mathrm{~mm}$
Figure 4. Metamorphosis of Res. 2 and Res. 4.

depends on the absolute temperature. Since the temperature decreases due to the expansion of the gas while the piston moves down, the speed of sound and the resonance frequencies decrease, too. (b) Due to piston motion the combustion chamber volume increases, i.e. the corresponding wavelengths of most resonances increase, so that again the frequencies decrease. In real combustion chambers both effects superimpose. Figure 5 shows the instantaneous frequencies of the resonances estimated by FEM simulations depending on crank angle and piston position, respectively. Obviously, all resonances except the first are frequency modulated - the frequencies decrease. Actually, the frequency of the first resonance slightly increases. Since for all simulations the speed of sound were constant, the frequency modulations were caused only by change of the chamber geometry. The frequency modulations correspond well to the shape of the resonances. If they contain axial oscillation components, there is a strong dependency on the piston position because piston position directly influences the wavelength of the standing wave. If they do not contain axial components, there is only a weak dependency, if any, except for small values of piston position, i.e. near top dead center. In that case the influence of the cylinder head causes a frequency modulation. Since the frequency modulation depends on the shape of the resonances it corresponds to the metamorphosis. E.g. Res. 4 contains no axial components until a piston position of about $40 \mathrm{~mm}$, see Figure 3 . Therefore, it is hardly frequency modulated in this interval. Between 40 $\mathrm{mm}$ and $68 \mathrm{~mm}$ the metamorphosis yields an axial component, see Figure 3 and 4 , corresponding to a strong frequency modulation, see Figure 5 . From $68 \mathrm{~mm}$ to bottom dead center there is again no axial component and therefore almost no frequency modulation. Another interesting point is that the frequencies of the first and second (from $68 \mathrm{~mm}$ to bottom dead center the fourth) resonances move closer together while the piston moves down. For large values of piston position the influence of the cylinder head decreases and the frequencies converge toward the frequency of the first circumferential resonance of a right cylinder. Similarly it can be shown that the frequency modulations can be approximated using the resonances of a right cylinder. E.g. for the fourth resonance between $40 \mathrm{~mm}$ and $68 \mathrm{~mm}$ the combustion chamber height equals approximately half of the wavelength. Therefore, the frequency modulation can be very well described by

$$
f(z) \approx \frac{c}{2(a+z)},
$$

where $c$ is the speed of sound, $z$ is the piston position, and $a$ takes into consideration the effective cylinder head height. 

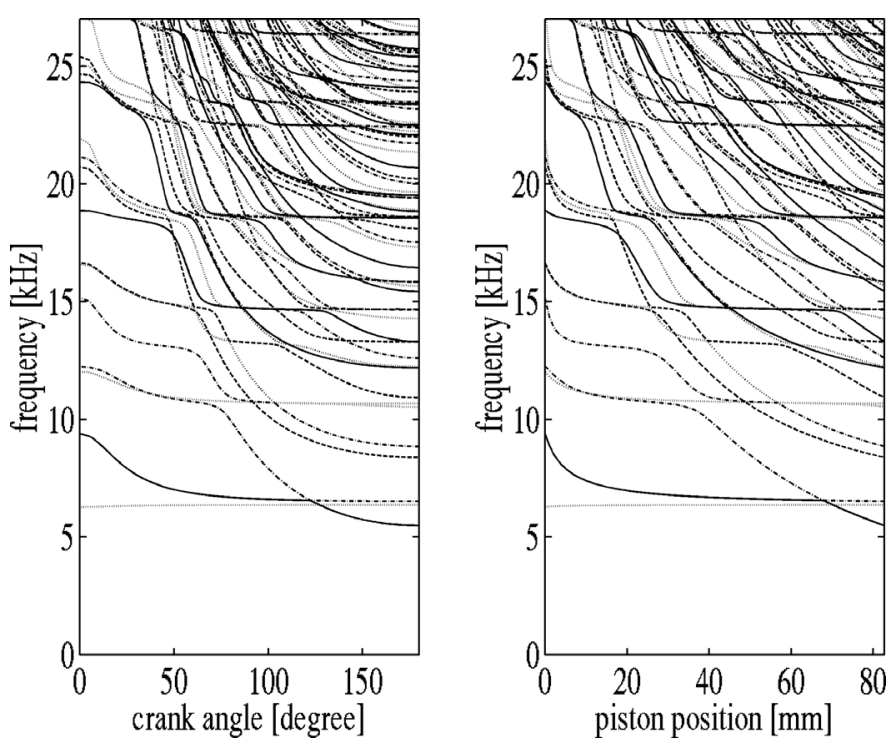

Figure 5. Instantaneous frequencies estimated by FEM simulations depending on crank angle (left) and on piston position (right).

As mentioned before, the speed of sound also influences the resonance frequencies. Since the distribution of pressure nodes and antinodes are determined only by the combustion chamber geometry, the frequency modulation of all resonances due to varying speed of sound is the same. If the speed of sound differs from the basic speed $c_{0}$ by a factor $c_{z}$, the frequencies of all resonances change by this factor. It is very difficult to estimate the speed of sound in a real combustion chamber using physical relations like the gas laws and adiabatic expansion of the gas because the necessary assumptions do not hold. Therefore, in this work the speed of sound was estimated using measured pressure data. From a high-resolution time-frequency representation the instantaneous frequency of the first resonance was estimate as $\hat{f}_{1}(\alpha)$, where $\alpha$ is crank angle. Then the speed of sound can be estimated by

$$
\hat{c}(\alpha)=\frac{\hat{f}_{1}(\alpha)}{f_{1}(\alpha)} c_{0}
$$

where $f_{1}(\alpha)$ is the instantaneous frequency estimated by FEM and $c_{0}$ is the speed of sound for the FEM simulations. The frequency of the $n$-th resonance can then be estimated by

$$
\hat{f}_{n}(\alpha)=\frac{\hat{c}(\alpha)}{c_{0}} f_{n}(\alpha)=\frac{\hat{f}_{1}(\alpha)}{f_{1}(\alpha)} f_{n}(\alpha)
$$

Figure 6 shows the Wigner-Ville spectra of pressure signals measured at $5500 \mathrm{rpm}$ by two pressure sensors at approximately sensor position 1 and 2, see Figure 1 . The Wigner-Ville spectrum provides a high-resolution time-frequency representation of multi-component signals, $[4,5]$. Dark regions indicate strong components. In order to stress weak components a logarithmic gray
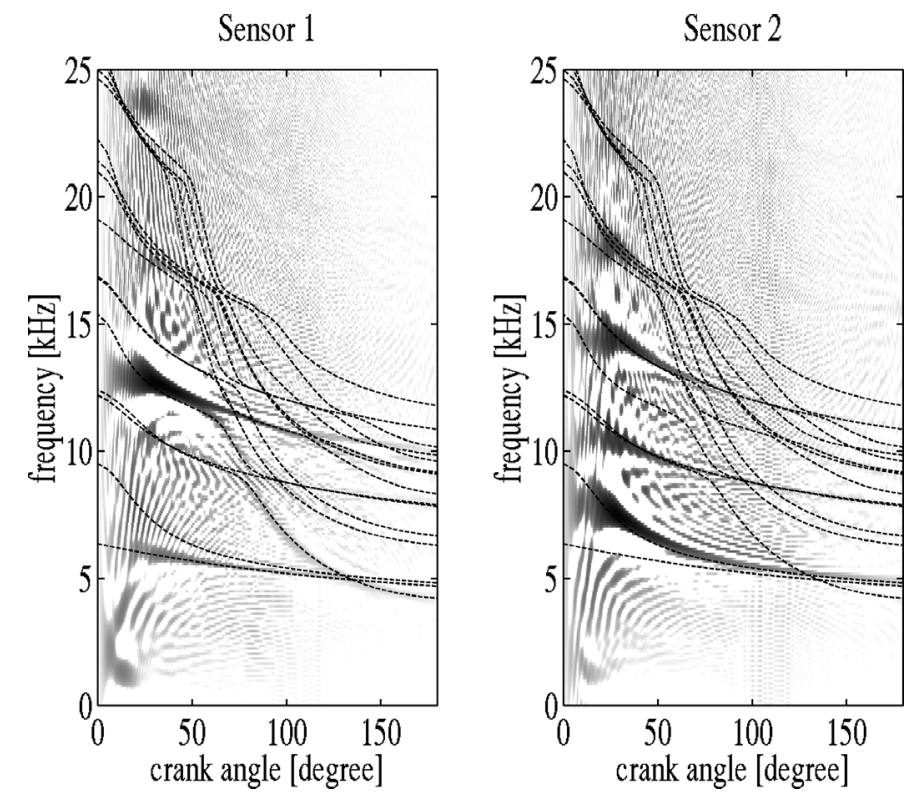

Figure 6. Estimated Wigner-Ville spectra of measured signals of two pressure sensors super-imposed by selected and corrected instantaneous frequencies estimated by FEM simulations.

scale was used. The spectra are superimposed by the instantaneous frequencies estimated by FEM simulations and corrected as described above. Obviously, the signals contain different components. This phenomenon will be discussed in the next section. Each component of the spectra corresponds to at least one FEM frequency. The spectrum of Sensor 1 mainly contains one component that corresponds to the fifth resonance until $50^{\circ}$ crank angle. Res. 1 is observed weakly and Res. 4 between $80^{\circ}$ and $130^{\circ}$ crank angle. Sensor 2 observes Res. 2, Res. 3 or 4 , and Res. 6 or 7 . The assignment of the fourth component of the spectrum is difficult because there are four resonances close together in the timefrequency domain.

\section{AMPLITUDE MODULATION}

The amplitudes of the oscillations in a real combustion chamber decrease because of heat losses, friction and gas expansion. These effects influence all resonances in the same way. But there is another aspect that causes an additional amplitude modulation due to pressure sensor position and resonance metamorphosis. As mentioned before the Wigner-Ville spectra of Figure 6 contain different components. Since the sensors observed the same combustions in the same combustion chamber, this can be only explained by their positions. Sensor 1 was mounted in the central spark plug. Most resonances have a pressure node on the cylinder axis, see Figure 2. Near top dead center only Res. 5 has a pressure antinode and should be well observable by Sensor 1. That is exactly what the spectrum of this sensor shows. In addition, Res. 1 is visible because the sensor position is slightly decentralized from the cylinder axis and Res. 1 is usually excited very strongly. Between $80^{\circ}$ and $130^{\circ}$ there is a component in the spectrum that corresponds to Res. 4 comparing the frequencies. This 
Res. 1
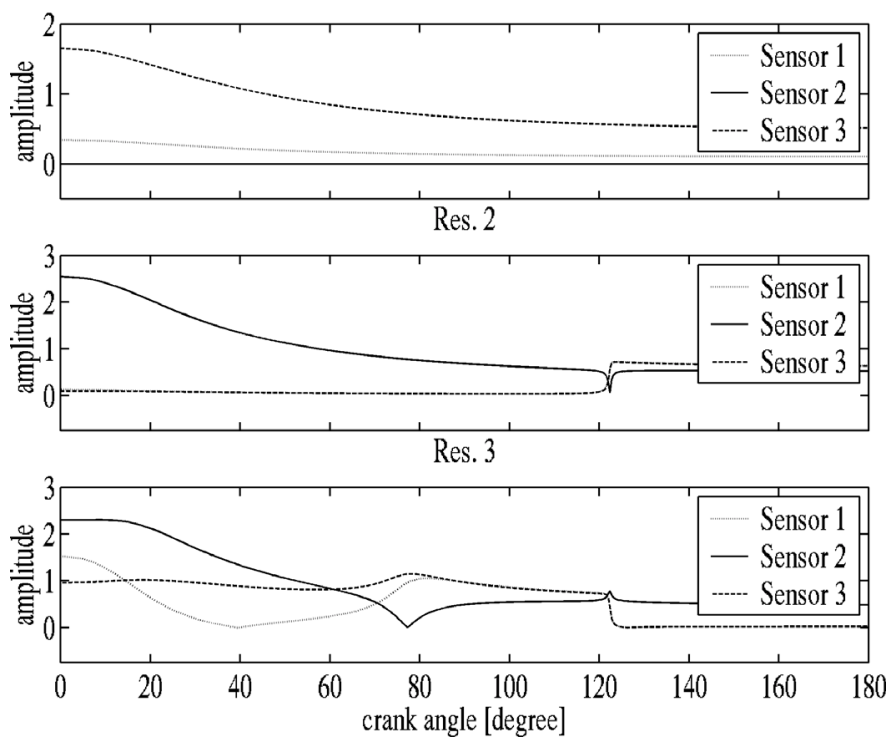

Figure 7. Amplitude modulation of Res. 1, 2, and 4 depending on the virtual pressure sensor position.

fits well to the metamorphosis of this resonance, see Figure 3. Near top dead center and near bottom dead center the resonance has a pressure node at the spark plug but meanwhile there is a pressure antinode. Therefore from Sensor 1's point of view Res. 4 is strongly amplitude modulated. The invisibility of most other resonances can be regarded as a kind of amplitude modulation, too. Figure 7 shows the amplitude modulation of Res. 1, 2, and 4 estimated by evaluating the FEM simulation results at the virtual pressure positions 1, 2, and 3, see Figure 1. Res.1 is observed best by Sensor 3. Its amplitude decreases but this amplitude modulation is caused by numerical effects. Sensor 1 observes this resonance with smaller amplitude and Sensor 2 does not notice this resonance at all. Res. 2 is visible only for Sensor 2 until $120^{\circ}$ crank angle. Then due to the metamorphosis of this resonance, all three sensors observe the resonance. The amplitude modulation of Res. 4 is more complex. The three sensors observe this resonance at top dead center. But Sensor 1 and Sensor 2 run through a pressure node at about $40^{\circ}$ crank angle and $80^{\circ}$ crank angle, respectively. From about $120^{\circ}$ crank angle this resonance is not visible for Sensor 3 any more.

\section{SENSOR CHARACTERISTIC}

The previous two sections have shown that the frequency modulation and the amplitude modulation of the resonances can be estimated using FEM simulations. In return the information can be used to predict the characteristic of a signal and its components measured by a pressure sensor at a certain position in the combustion chamber. The intensity of excitation of the resonances is random and can not be predicted, of course. The frequency modulation due to varying speed of sound and the amplitude modulation due to heat losses and friction are neither predicted here. But the
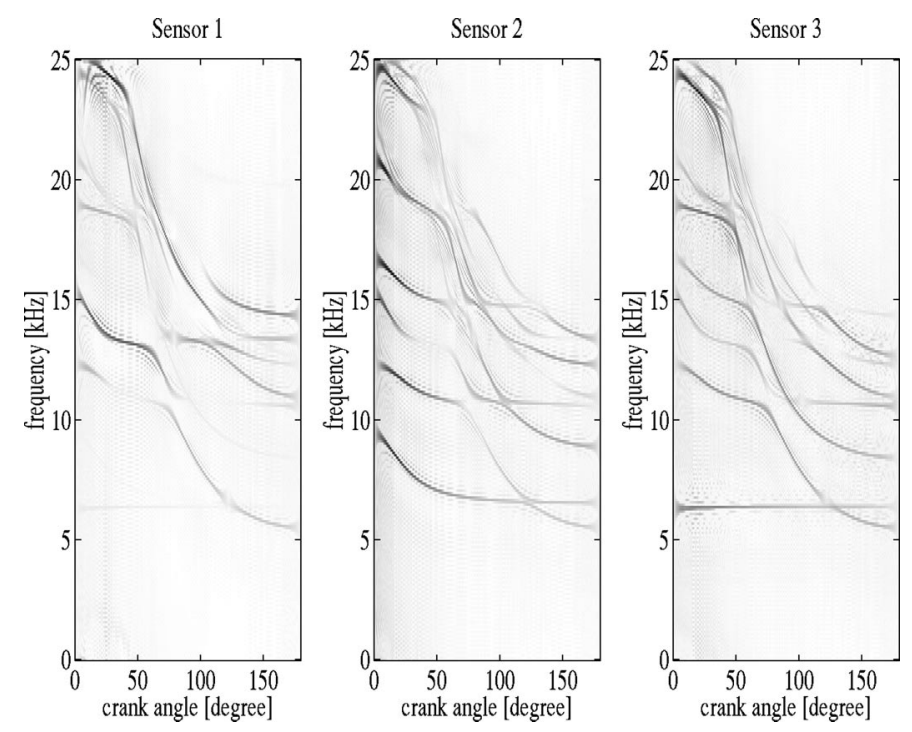

Figure 8. Sensor characteristic of Sensor 1, 2, and 3.

frequency modulation and amplitude modulation due to metamorphosis contains a lot of information with respect to assess a pressure sensor position. The Wigner-Ville spectrum of such a predicted signal, called sensor characteristic, yields a simple tool to compare several pressure sensor positions. Figure 8. shows the characteristic of the three virtual sensors. The characteristic of Sensor 1 and 2 are quite similar to the Wigner-Ville spectra of the measured data in Figure 6.

\section{INFLUENCE OF KNOCK ORIGIN}

It is well known that the excitation of the resonances is random and hardly correlated from combustion to combustion. The cause is the random character of the combustion process and of knock. In this section we will show that especially the origin of knock may have strong influence on the excitation.

MODEL: In order to simulate the explosive character of knock transient simulations were performed. The combustion chamber model equals that of the modal analysis at a piston position of $z=1.8 \mathrm{~mm}$ corresponding to $15^{\circ}$ crank angle. This piston position was chosen because knock occurs near this point. The speed of sound again was set to $c_{0}=1000 \mathrm{~m} / \mathrm{s}$. A certain set of nodes got an initial pressure level simulating the pressure step caused by knock. With this initial condition the pressure distribution at time $t=\Delta$, then $t=2 \Delta$ and so on was calculated. Altogether $N=4000$ steps were simulated at a step size of $\Delta=0.5 \mu \mathrm{s}$. This means that a time interval of $N \Delta=2 \mathrm{~ms}$ was considered. During this time interval the crank angle would rotate to $17^{\circ}$ at $1000 \mathrm{rpm}$ which means that the piston would move down to $z=2.3 \mathrm{~mm}$. But for simulation simplicity the piston motion was neglected. This approximation is justified because the piston velocity at $15^{\circ}$ crank angle and $1000 \mathrm{rpm}$ is about 

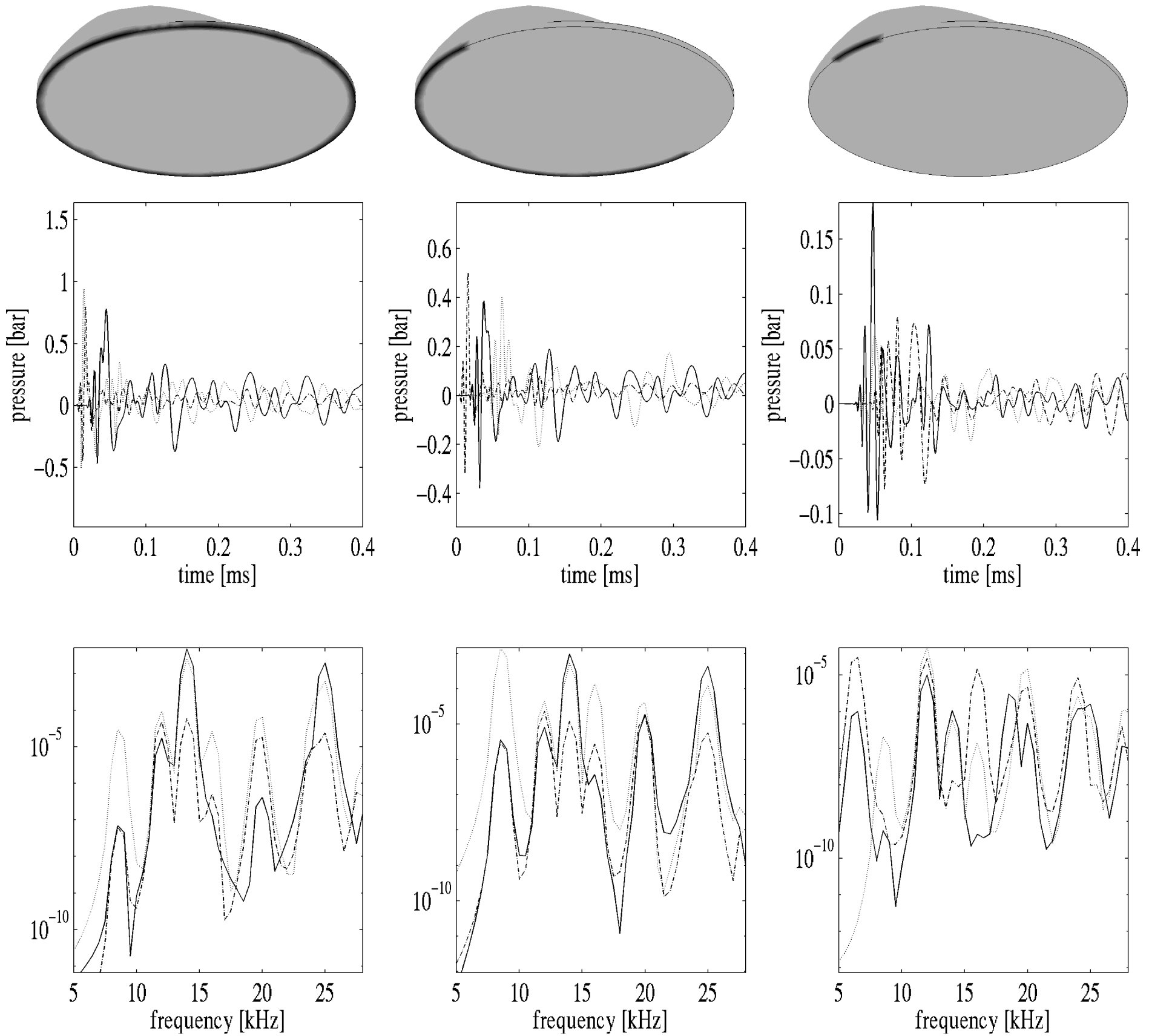

Figure 9. Transient analysis for different kinds of excitation (top), according time signals (middle) and according periodograms (bottom). Solid lines mark Sensor 1, dotted lines Sensor 2, and dash-dotted lines Sensor 3.

$0.5 \mathrm{~m} / \mathrm{s}$ and therefore much slower as speed of sound $(1000 \mathrm{~m} / \mathrm{s})$. This means that even the lowest resonance frequency is high enough so that the piston hardly moves during an oscillation period. Furthermore, the modal analyses together with measured data have shown that resonances containing also axial components are stable even at high engine speeds and high piston velocities. Therefore, only the excitation of the resonances is crucial.

Theoretically the time signals of all nodes could be evaluated. But the amount of information is infeasible. So this investigations limit to the signals of the three virtual pressure sensors marked in Figure 1.
RESULTS: Figure 9 demonstrates three different kinds of excitation. The top line shows the combustion chamber with the initial pressure impulse marked as dark gray. The first example is an annular excitation. The end gas that is assumed to burn by knock is considered as a ring above the piston at the cylinder wall. The second example is a half ring above the piston on the outlet side of the combustion chamber. The third example considers a small excitation region at one side of the cylinder.

Figure 10 shows the propagating pressure wave for the first example at several time instants. Here, dark gray generally means high pressure but each image is scaled relatively between highest and lowest pressure values. Therefore, it is difficult to compare the amplitudes of 


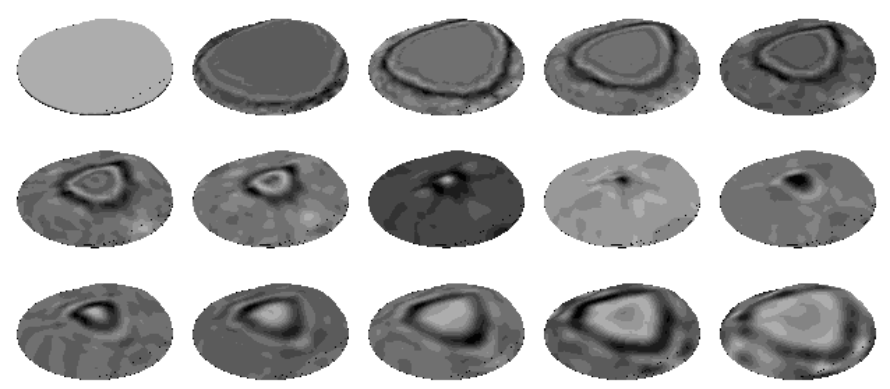

Figure 10. Instantaneous pressure distribution at time instants $t=0,10 \Delta, 20 \Delta, \ldots, 140 \Delta$ in case of the annular excitation.

different images. The annular excitation causes an annular pressure wave front propagating towards the cylinder axis yielding a pressure peak when they meet there. Figure 9, middle, shows the time signals of the virtual pressure sensors in the interval from 0 to $0.4 \mathrm{~ms}$. Depending on the excitation location and sensor position there is a delay between excitation instant and the time when the sensors get signals. This delay corresponds to the distance between excitation and sensor and the speed of sound. The delay can be observed best in the third example. After the sensors have got the first signals they observe some kinds of oscillation. In order to investigate the oscillations the signals were transferred in frequency domain by estimating their periodograms, see Figure 9, bottom. Table 1 contains the frequencies of the resonances at piston position $z=1.8 \mathrm{~mm}$ in order to be able to assign the periodogram peaks to the combustion chamber resonances. The strongest component in the first example that is observed by Sensor 1 has a frequency of about $14 \mathrm{kHz}$. This corresponds well to Res. 5. Comparing to Figure 2 this resonance has a pressure antinode on the cylinder axis and that is why Sensor 1 can observe the resonance well. Figure 10 demonstrates the excitation of Res. 5 too. Sensor 2 samples this resonance also with high amplitude, because its position is near a pressure antinode too. Sensor 3 observes this resonance worse due to its position. The second resonance is received well by Sensor 2 at about $8.5 \mathrm{kHz}$. Both other sensors receive this resonance too, but their amplitudes are very low. In the second example Res. 2 is the strongest component observed best by Sensor 2 . Res. 5 again was excited strongly well received by Sensor 1 and 2, similar to the first example. In the third example Res. 1 and most likely Res. 4, 7 and 10 are strongly excited. While it is easy to identify Res. 1 , it is more difficult for the other resonances because there are more resonance with similar frequencies. But the excitation in the third example takes place in pressure antinodes of the mentioned resonances. Other resonances like Res. 3, 6, and 9 have a pressure node at the location of excitation. So they are not or only weakly excited. Although Res. 1 is strongly excited according to Sensor 3, this resonance is not visible in the periodogram of Sensor 2 at all because Sensor 2 is perfectly located in

\begin{tabular}{|c|c||c|c|}
\hline Res. & Freq. $[\mathrm{kHz}]$ & Res. & Freq. $[\mathrm{kHz}]$ \\
\hline \hline 1 & 6.31 & 9 & 19.80 \\
\hline 2 & 8.63 & 10 & 19.97 \\
\hline 3 & 11.70 & 11 & 20.26 \\
\hline 4 & 11.94 & 12 & 23.89 \\
\hline 5 & 14.04 & 13 & 23.94 \\
\hline 6 & 15.99 & 14 & 24.00 \\
\hline 7 & 16.03 & 15 & 24.13 \\
\hline 8 & 18.69 & 16 & 24.92 \\
\hline
\end{tabular}

Table 1. Resonance frequencies at piston position $z=1.8 \mathrm{~mm}$.

a pressure node of Res. 1. The excitations in these examples are quite simple and arbitrary but they show that a random knock origin may cause the random excitation of the resonances. If so, the periodogram of the sensor signals can be regarded as a kind of finger print of the kind of excitation. Vice versa, it could be possible to determine the knock origin from the periodogram.

\section{CONCLUSION}

The FEM approach has been successfully applied to model combustion chamber resonances. The frequency and amplitude modulations of the resonances corresponding to their metamorphosis were estimated and a sensor characteristic to assess pressure sensor positions was proposed. Simulation results fit well results of measured data using Wigner-Ville spectra as highresolution time-frequency representation. Applying transient analyses it was demonstrated that the random excitation of the resonances is very likely caused by the random origin of knock.

\section{REFERENCES}

1. Draper, C. S., "Pressure Wave Accompanying Detonation in the Internal Combustion Engine", Journal of the Aeronautic Sciences, Vol. 5, No. 6, pp. 219-226, 1938.

2. Hickling, R., Feldmaier, D. A., Chen, F. H. K., Morel, J. S., "Cavity Resonances in Engine Combustion Chambers and Some Applications", J. Acoust. Soc. Am., 73 (4), pp. 1170-1178, 1983.

3. Scholl, D., Davis, C. Russ, S. Barash, T., "The Volume Acoustic Modes of Spark-Ignited Internal Combustion Chambers", SAE Paper 980893, 1998.

4. Martin, W., Flandrin, P., "Wigner-Ville-Spectral Analysis of Nonstationary Processes", IEEE Trans. on ASSP, 33 (6), pp. 1461-1470, 1985.

5. Böhme, J. F., König, D., "Statistical Processing of Car Engine Signals for Combustion Diagnosis”, Proc. IEEE $7^{\text {th }}$ Workshop on Statistical Signal and Array Processing, pp. 369-374, invited paper, 1994. 